\title{
Biliary scintiscan had high sensitivity and specificity for predicting pathological findings in the common bile duct
}

Mathur SK, Soonawalla ZF, Shah SR, et al. Role of biliary scintiscan in predicting the need for cholangiography. Br J Surg $2000 \mathrm{Feb} ; 87: 181-5$.

QUESTION: In patients with symptomatic gallstone disease, can biliary scintiscan predict the presence of pathological findings in the common bile duct (CBD)?

Design

A blinded comparison of scintiscan and ultrasonography alone or combined with clinical or standard criteria (history of jaundice or acute pancreatitis, increased serum bilirubin and alkaline phosphatase concentrations, and visualisation of a stone or presence of dilated bile ducts on ultrasonography) and modified criteria (jaundice within the past $3 \mathrm{mo}$, increased serum bilirubin and alkaline phosphatase concentrations, and visualisation of a stone or presence of dilated bile ducts on ultrasonography).

Setting

A tertiary referral centre in Bombay, India.

Patients

75 consecutive patients (mean age 46 y, $61 \%$ women) with symptomatic gallstone disease. Patients with acute cholecystitis, acute pancreatitis, or cholangitis were excluded.

\section{Description of tests and diagnostic standard}

Biliary scintigraphy was done using intravenous injection of $5 \mu \mathrm{Ci}{ }^{99 m} \mathrm{Tc}$ radio labelled mebrofenin with a recording at baseline and at 1 and 2 hours. Reading of recordings was blinded using predetermined criteria (standard and modified) for pathological findings in the CBD. Positive ultrasonographic criteria were visualisation of a CBD stone, presence of intrahepatic bile duct dilatation, or common hepatic duct or CBD size $>7$ $\mathrm{mm}$. The diagnostic standard was endoscopic or preoperative cholangiography; if calculi were found, endoscopic sphincterotomy or open surgical exploration of the CBD was done.

Diagnostic findings and tests for predicting common bile duct (CBD) pathology in patients with symptomatic gallstone disease*

\begin{tabular}{llcll} 
Diagnostic findings and tests & Sensitivity & Specificity & +LR & -LR \\
Abnormal findings on biliary scintigraphy & $93 \%$ & $94 \%$ & 15 & 0.07 \\
\hline CBD $>9$ mm with ultrasonography & $63 \% \dagger$ & $100 \% \dagger$ & Infinity & 0.4 \\
\hline CBD stone with ultrasonography & $46 \% \dagger$ & $100 \% \dagger$ & Infinity & 0.5 \\
\hline Abnormal bile duct with ultrasonography & $67 \% \dagger$ & $96 \%$ & 17 & 0.3 \\
\hline All standard criteria combined & $89 \%$ & $48 \% \dagger$ & 1.7 & 0.23 \\
\hline Modified standard criteria & $89 \%$ & $71 \% \dagger$ & 3 & 0.2 \\
\hline Ultrasonography and scintiscan & $96 \%$ & $98 \%$ & 48 & 0.04 \\
\hline
\end{tabular}

*Likelihood ratios defined in glossary and calculated from data in article.

$\dagger$ Statistically different when compared with biliary scintigraphy $(p \leqslant 0.05)$.
Main outcome measures

Sensitivity and specificity of features of biliary scintiscan, ultrasonography, and clinical criteria for predicting pathological findings in the CBD.

\section{Main results}

Sensitivity and specificity for biliary scintiscan alone and combined with ultrasonography were high (table). The table lists the sensitivity and specificity of other features or variables.

\section{Conclusion}

Sensitivity and specificity for biliary scintiscan alone and combined with ultrasonography were high.

\section{COMMENTARY}

Cholangiography is considered the diagnostic standard in detecting CBD stones. Endoscopic retrograde and perioperative (intraoperative) cholangiography are 2 widely used methods. Both, however, are invasive and entail some risk. Finding a non-invasive or risk free method of confirming or excluding CBD stones has always been frustrating. Mathur $e t$ al have evaluated biliary scintigraphy and other non-invasive methods and criteria. Biliary scintigraphy was found to be superior to many of the non-invasive criteria used by the authors.

The best use of biliary scintigraphy to diagnose choledocolithiasis is probably in patients at high risk for cholangiography related complications and with low probability for choledocolithiasis. In such patients, more information is needed to justify the risks and costs of cholangiography, especially if experts who can do the procedures are not readily available.

Potential criticisms of the study include a lack of clearly stated patient inclusion criteria, a lack of clearly stated criteria for biliary obstruction as seen on scintigraphy, and a methods section that requires several readings to be understood. Finally, all of the tests evaluated are highly operator dependent. Individual expertise should be considered when deciding to apply the results of this study.

The authors have re-explored and given new life to a widely available, safe, and relatively inexpensive tool. They are to be commended. Their results should spur others to investigate further the use of biliary scintigraphy.

William B Silverman, MD University of Iowa Hospitals and Clinics Iowa City, Iowa, USA 